\title{
Effect of Non-Homogeneity on Thermally Induced Vibration of Orthotropic Visco-Elastic Rectangular Plate of Linearly Varying Thickness
}

\author{
Arun Kumar Gupta, Pooja Singhal \\ Department of Mathematics, M.S. College, Saharanpur, Uttar Pradesh, India \\ E-mail: guptaarunnitin@yahoo.co.in,poojaacad@yahoo.in \\ Received August 3, 2010; revised August 30, 2010; accepted September 3, 2010
}

\begin{abstract}
The analysis presented here is to study the effect of non-homogeneity on thermally induced vibration of orthotropic visco-elastic rectangular plate of linearly varying thickness. Thermal vibrational behavior of non -homogeneous rectangular plates of variable thickness having clamped boundary conditions on all the four edges is studied. For non-homogeneity of the plate material, density is assumed to vary linearly in one direction. Using the method of separation of variables, the governing differential equation is solved. An approximate but quite convenient frequency equation is derived by using Rayleigh-Ritz technique with a two-term deflection function. Time period and deflection at different points for the first two modes of vibration are calculated for various values of temperature gradients, non-homogeneity constant, taper constant and aspect ratio. Comparison studies have been carried out with non-homogeneous visco-elastic rectangular plate to establish the accuracy and versatility.
\end{abstract}

Keywords: Non-Homogeneous, Orthotropic, Visco-Elastic, Variable Thickness, Rectangular Plate, Vibration, Thermal Gradient

\section{Introduction}

Thermal effect on vibration of non-homogenous viscoelastic plates are of great interest in the field of engineering such as for better designing of gas turbines, jet engine, space craft and nuclear power projects, where metals and their alloys exhibits visco-elastic behavior. Therefore, for these reason such structures are exposed to high intensity heat fluxes and thus material properties undergo significant changes, in particular the thermal effect on the modules of elasticity of the material can not be taken as negligible.

Plates of variable thickness have been extensively used in Civil, Electronic, Mechanical, Aerospace and Marine Engineering applications. The practical importance of such plates has made vibration analysis essential especially since the vibratory response needs to be accurately determined in design process in order to avoid resonance excited by internal or external forces.

Visco-elastic, as its name implies, is a generalization of elasticity and viscosity. The ideal linear elastic element is the spring. When a tensile force is applied to it, the increase in the distance between its two ends is proportional to the force. The ideal linear viscous element is the dashpot.

The plate type structural components in aircraft and rockets have to operate under elevated temperatures that cause non-homogeneity in the plate material i.e. elastic constants of the materials becomes functions of space variables. In an up-to-date survey of literature, authors have come across various models to account for non-homogeneity of plate materials proposed by researchers dealing with vibration but none of them consider non-homogeneity with thermal effect on orthotropic visco-elastic plates.

Free vibration of visco-elastic orthotropic rectangular plates was discussed by Sobotka [1].Gupta and Khanna [2] discussed vibration of viscoelastic rectangular plate with linearly thickness variations in both directions. Leissa's monograph [3] contains an excellent discussion of the subject of vibrating plates with elastic edge support. Several authors $[4,5]$ have studied the thermal effect on vibration of homogeneous plates of variable thickness but no one considered thermal effect on vibra- 
tion on non-homogeneous rectangular plates of varying thickness. Tomar and Gupta [6-8] solved the vibration problem of orthotropic rectangular plate of varying thickness subjected to a thermal gradient. Gupta, Lal and Sharma [9] discussed the vibration of non-homogeneous circular plate of nonlinear thickness variation by a quadrature method. Gupta, Johri and Vats [10] solved the problem of thermal effect on vibration of non-homogeneous orthotropic rectangular plate having bi-directional parabolically varying thickness. Gupta, Kumar and Gupta [11] studied the vibration of visco-elastic orthotropic parallelogram plate with a linear variation of thickness. Recently, Gupta and Kumar [12] solved the vibration problem of non-homogeneous visco-elastic rectangular plate of linearly varying thickness subjected to linearly thermal effect. Free vibration of a clamped visco-elastic rectangular plate having bi-direction exponentially varying thickness were studied by Gupta, Khanna and Gupta [13] .Gupta, Aggarwal, Gupta, Kumar and Sharma [14] discussed the non-homogeneity on free vibration of orthotropic visco-elastic rectangular plate of parabolic varying thickness. Subsequent review article of Bhasker and Kaushik [15] are best source for problems involving rectangular plates fall into three distinct categories: 1) plates with all edges simply supported; 2) plates with a pair of opposite edges simply supported; 3) plates which do not fall into any of the above categories.

Rectangular plates have wide applications in civil structures, electrical engineering, marine industry and mechanical engineering. The dynamic characteristics of rectangular plates are important to engineering designs. An analysis is presented in this paper is to study the effect of non-homogeneity on thermally induced vibration of orthotropic visco-elastic rectangular plate of linearly varying thickness. It is clamped supported on all the four edges. The assumption of small deflection and linear orthotropic visco-elastic properties are made. It is further assumed that the visco-elastic properties of the plates are of the Kelvin type. For this the material constants of alloy 'Duralium' is used for the calculation of numerical values. Time period and deflection for the first two modes of vibration are calculated for the various values of thermal gradients, non-homogeneity constant, aspect ratio and taper constant.

\section{Analysis}

The equation of motion of a visco-elastic orthotropic rectangular plate of variable thickness may be written in the form, as by Sobotka [1]

$$
\frac{\partial^{2} M_{x}}{\partial x^{2}}+2 \frac{\partial^{2} M_{x y}}{\partial x \partial y}+\frac{\partial^{2} M_{y}}{\partial y^{2}}=\rho h \frac{\partial^{2} w}{\partial t^{2}}
$$

Here $M_{x}, M_{y}$ and $M_{x y}$ are moments per unit length of plate, $\rho$ is mass per unit volume, $h$ is thickness of plate and $w$ is displacement at time $t$.

The expression for $M_{x}, M_{y}, M_{x y}$ are given by

$$
\begin{aligned}
& M_{x}=-\tilde{D}\left[D_{x} \frac{\partial^{2} w}{\partial x^{2}}+D_{1}^{\prime} \frac{\partial^{2} w}{\partial y^{2}}\right] \\
& M_{y}=-\tilde{D}\left[D_{1}^{\prime} \frac{\partial^{2} w}{\partial x^{2}}+D_{y} \frac{\partial^{2} w}{\partial y^{2}}\right] \\
& M_{x y}=-2 \tilde{D} D_{x y} \frac{\partial^{2} w}{\partial x \partial y}
\end{aligned}
$$

where

$$
D_{x}=\frac{E_{x} h^{3}}{12\left(1-v_{x} v_{y}\right)}
$$

is called the flexural rigidity of the plate in $x$-direction,

$$
D_{y}=\frac{E_{y} h^{3}}{12\left(1-v_{x} v_{y}\right)}
$$

is called the flexural rigidity of the plate in $y$-direction,

$$
D_{x y}=\frac{G_{x y} h^{3}}{12}
$$

is called the torsion rigidity, and

$$
D_{1}^{\prime}=v_{x} D_{y}\left(=v_{y} D_{x}\right)
$$

Here $\tilde{\mathrm{D}}$ is Rheological operator and $E_{x} \& E_{y}$ are the modules of elasticity in $x$ - and $y$-direction respectively, $v_{x}$ and $v_{y}$ are the Poisson ratios \& $G_{x y}$ is the shear modulus.

Taking deflection $\mathrm{w}$ as a product of two functions as:

$$
w=w(x, y, t)=W(x, y) T(t)
$$

where $W(x, y)$ is the function of coordinates in $x, y$ and $T$ $(t)$ is a time function.

Using Equation (3) in Equations (1) \& (2) and then equating both sides of equation comes to a constant, say $p^{2}$, one gets two separate differential equations as follows:

$$
\begin{aligned}
& {\left[D_{x} \frac{\partial^{4} W}{\partial x^{4}}+D_{y} \frac{\partial^{4} W}{\partial y^{4}}+2 H \frac{\partial^{4} W}{\partial x^{2} \partial y^{2}}+2 \frac{\partial H}{\partial x} \frac{\partial^{3} W}{\partial x \partial y^{2}}\right.} \\
& +2 \frac{\partial H}{\partial y} \frac{\partial^{3} W}{\partial x^{2} \partial y}+2 \frac{\partial D_{x}}{\partial x} \frac{\partial^{3} W}{\partial x^{3}}+2 \frac{\partial D_{y}}{\partial y} \frac{\partial^{3} W}{\partial y^{3}} \\
& +\frac{\partial^{2} D_{x}}{\partial x^{2}} \frac{\partial^{2} W}{\partial x^{2}}+\frac{\partial^{2} D_{y}}{\partial y^{2}} \frac{\partial^{2} W}{\partial y^{2}}+\frac{\partial^{2} D_{1}^{\prime}}{\partial x^{2}} \frac{\partial^{2} W}{\partial y^{2}} \\
& \left.+\frac{\partial^{2} D_{1}^{\prime}}{\partial y^{2}} \frac{\partial^{2} W}{\partial x^{2}}+4 \frac{\partial^{2} D_{x y}}{\partial x \partial y} \frac{\partial^{2} W}{\partial x \partial y}\right]-\rho h p^{2} W=0
\end{aligned}
$$


and

$$
\ddot{T}+p^{2} \tilde{D} T=0
$$

where

$$
H=D_{1}^{\prime}+2 D_{x y}
$$

Equation (4) is a differential equation of motion for orthotropic rectangular plate of variable thickness and (5) is a differential equation of time functions of free vibration of viscoelastic rectangular orthotropic plate.

The temperature dependence of the modulus of elasticity for orthotropic materials is given by

$$
\begin{aligned}
& E_{x}=E_{1}(1-\gamma \tau) \\
& E_{y}=E_{2}(1-\gamma \tau) \\
& G_{x y}=G_{0}(1-\gamma \tau)
\end{aligned}
$$

and temperature distribution along the length i.e. in the $x$-direction,

$$
\tau=\tau_{o}(1-x / a)
$$

where $\tau$ denotes the temperature excess above the reference temperature at any point at distance $x / a$ and $\tau_{0}$ denotes the temperature excess above reference temperature at the end, i.e. for $x=0$. Here $E_{1}$ and $E_{2}$ are values of the Young's moduli respectively along the $x$ and $y$ axis at the reference temperature i.e. at $\tau=0$

The modulus variation (6) in view of expressions (7) becomes

$$
\begin{aligned}
& E_{x}(x)=E_{1}[1-\alpha(1-x / a)] \\
& E_{y}(x)=E_{2}[1-\alpha(1-x / a)] \\
& G_{x y}(x)=G_{0}[1-\alpha(1-x / a)]
\end{aligned}
$$

where $\alpha=\gamma \tau_{o}(0 \leq \alpha<1)$, known as thermal gradient.

The expression for the strain energy $V$ and Kinetic energy $P$ in the plate are [3]

$$
\begin{aligned}
& \frac{1}{2} \int_{0}^{a} \int_{0}^{b}\left[D_{x}\left(W_{, x x}\right)^{2}+D_{y}\left(W_{, y y}\right)^{2}+2 D_{1} W_{, x x} W_{, y y}\right. \\
& \left.+4 D_{x y}\left(W_{, x y}\right)^{2}\right] d x d y \\
& P=\frac{1}{2} p^{2} \int_{0}^{a} \int_{0}^{b} \rho h W^{2} d x d y
\end{aligned}
$$

The thickness and density varies linearly in the $\mathrm{x}$-direction only, so let us assume

$$
h=h_{o}(1+\beta x / a)
$$

and $\rho=\rho_{o}\left(1+\alpha_{1} x / a\right)$

where $\beta$ is the taper constant and $\alpha_{1}$ is non-homogeneity constant.

\section{Solution and Frequency Equation}

To find the solution, we use Rayleigh-Ritz technique. In this method, one requires maximum strain energy be equal to the maximum Kinetic energy. So, it is necessary for the problem under consideration that

$$
\delta(V-P)=0
$$

for arbitrary variations of $\mathrm{W}$ satisfying relevant geometrical boundary conditions are

$$
\begin{aligned}
& W=W_{, x}=0 \text { at } x=0, a \\
& W=W_{, y}=0 \text { at } y=0, b
\end{aligned}
$$

and the corresponding two term deflection function is taken as [6]

$$
\begin{aligned}
& W=[(x / a)(y / b)(1-x / a)(1-y / b)]^{2} \\
& {\left[A_{1}+A_{2}(x / a)(y / b)(1-x / a)(1-y / b)\right]}
\end{aligned}
$$

The non-dimensional variables are

$$
\begin{aligned}
& X=x / a, Y=y / b, \bar{W}=W / a, \bar{h}=h / a, \\
& \bar{\rho}=\rho / a \\
& E_{1}^{*}=E_{1} /\left(1-v_{x} v_{y}\right), E_{2}^{*}=E_{2} /\left(1-v_{x} v_{y}\right) \\
& E^{*}=v_{x} E_{2}^{*}=v_{y} E_{1}^{*}
\end{aligned}
$$

By using Equations (8), (11) and (12) in (9) and (10), one gets

$$
\left.P=\frac{1}{2} \rho_{o} p^{2} \overline{h_{o}} a^{5} \int_{0}^{1} \int_{0}^{b / a}\left[\left(1+\alpha_{1} X\right)(1+\beta X) \bar{W}^{2}\right] d X d Y\right)
$$

and

$$
\begin{aligned}
& V=R \int_{0}^{1} \int_{0}^{b / a}\left[\{1-\alpha(1-X)\}(1+\beta X)^{3}\right. \\
& \left\{\left(\bar{W}_{, X X}\right)^{2}+\left(E_{2}^{*} / E_{1}^{*}\right)\left(\bar{W}_{, Y Y}\right)^{2}+\right. \\
& \left(2 E^{*} / E_{1}^{*}\right) \bar{W}_{, X X} \bar{W}_{, Y Y}+ \\
& \left.\left.\left(4 G_{o} / E_{1}^{*}\right)\left(\bar{W}_{, X Y}\right)^{2}\right\}\right] d X d Y
\end{aligned}
$$

where $R=\frac{1}{2}\left(E_{1}^{*} \bar{h}^{3} / 12\right) a$

On substituting the values of $P$ and $V$ from Equations (18) and (19) in Equation (13), we get

$$
\left(V_{1}-\lambda^{2} p^{2} P_{1}\right)=0
$$

$$
\begin{aligned}
& V=R \int_{0}^{1} \int_{0}^{b / a}\left[\{1-\alpha(1-X)\}(1+\beta X)^{3}\right. \\
& \left\{\left(\bar{W}_{, X X}\right)^{2}+\left(E_{2}^{*} / E_{1}^{*}\right)\left(\bar{W}_{, Y Y}\right)^{2}+\right. \\
& \left(2 E^{*} / E_{1}^{*}\right) \bar{W}_{, X X} \bar{W}_{, Y Y}+ \\
& \left.\left.\left(4 G_{o} / E_{1}^{*}\right)\left(\bar{W}_{, X Y}\right)^{2}\right\}\right] d X d Y
\end{aligned}
$$




$$
P_{1}=\int_{0}^{1} \int_{0}^{b / a}\left[\left\{\left(1+\alpha_{1} X\right)(1+\beta X) \bar{W}^{2}\right] d X d Y\right.
$$

where $\lambda^{2}=\frac{12 a^{4} \rho_{o}}{E_{1}^{*}{\overline{h_{o}}}^{2}}$

Equation (21) involves the unknown $A_{1}$ and $A_{2}$ arising due to the substitution of $W(x, y)$ from Equation (15). These two constants are to be determined from Equation (21) as follows:

$$
\frac{\partial}{\partial A_{n}}\left(V_{1}-\lambda^{2} p^{2} P_{1}\right)=0
$$

where $n=1,2$

On simplifying (25) we get

$$
b_{n 1} A_{1}+b_{n 2} A_{2}=0
$$

where $n=1,2, b_{n 1}, b_{n 2}$ involves parametric constants and the frequency parameter p. For a non-trivial solution, the determinant of the coefficient of Equation (26) must be zero. So, we get the frequency equation as

$$
\left|\begin{array}{ll}
b_{11} & b_{12} \\
b_{21} & b_{22}
\end{array}\right|=0
$$

On solving Equation (27) one gets a quadratic equation in $p^{2}$, which gives two values of $p^{2}$. On substituting the value of $A_{1}=1$, by choice, in Equation (15) one get $A_{2}=-b_{11} / b_{12}$ and hence $W$ becomes:

$$
\begin{aligned}
W= & {\left[X Y \frac{a}{b}(1-X)\left(1-Y \frac{a}{b}\right)\right]^{2} } \\
& {\left[1+\left(-\frac{b_{11}}{b_{12}}\right) X Y\left(\frac{a}{b}\right)(1-X)\left(1-Y \frac{a}{b}\right)\right] }
\end{aligned}
$$

\section{Time Functions of Vibration of Viscoelastic Plates}

The expression for Time function of free vibrations of visco-elastic plates of variable thickness can be derived from Equation (5) that depends upon visco-elastic operator $\tilde{D}$ and which for Kelvin's Model can be taken as:

$$
\tilde{D} \equiv\left\{1+\left(\frac{\eta}{G}\right)\left(\frac{d}{d t}\right)\right\}
$$

where $\eta$ is visco-elastic constant and $G$ is shear modulus. Taking temperature dependence of viscoelastic constant $\eta$ and shear modulus $G$ is the same form as that of Young's moduli, we have

$$
\begin{aligned}
& G(\tau)=G_{0}\left(1-\gamma_{1} \tau\right), \\
& \eta(\tau)=\eta_{0}\left(1-\gamma_{2} \tau\right)
\end{aligned}
$$

where $G_{0}$ is shear modulus and $\eta_{0}$ is visco-elastic constant at some reference temperature i.e. at $\tau=0, \gamma_{1}$ and $\gamma_{2}$ are slope variation of $\tau$ with $G$ and $\eta$ respectively. Substituting the value of $\tau$ from Equation (7) and using Equation (16) in Equation (30), one gets:

$$
G=G_{0}\left[1-\alpha_{5}(1-X)\right]
$$

where $\alpha_{5}=\gamma_{1} \tau_{0}, 0 \leq \alpha_{5}<1$

and $\eta=\eta_{0}\left[1-\alpha_{4}(1-X)\right]$

where $\alpha_{4}=\gamma_{2} \tau_{0}, 0 \leq \alpha_{4}<1$

Here $\alpha_{4}$ and $\alpha_{5}$ are thermal constants.

Substituting Equations (29) and (31) in Equation (5), one gets:

$$
\ddot{T}+p^{2} k \dot{T}+p^{2} T=0
$$

Where $k=\frac{\eta}{G}=\frac{\eta_{0}\left[1-\alpha_{4}(1-X)\right]}{G_{0}\left[1-\alpha_{5}(1-X)\right]}$

Equation (32) is a second order differential equation in time function $T$. The solution of which comes out to be

$$
T(t)=e^{a_{1} t}\left[C_{1} \cos b_{1} t+C_{2} \sin b_{1} t\right]
$$

where $a_{1}=-p^{2} k / 2$,

$$
b_{1}=p \sqrt{1-\left(\frac{p k}{2}\right)^{2}}
$$

where $C_{1}$ and $C_{2}$ are constants of integration, which can be determined easily from initial conditions of the plate.

Let us take initial conditions as

$$
T=1 \text { and } \dot{T}=0 \text { at } t=0
$$

Using Equation (36) in Equation (34), we have $C_{1}=1$ and

$$
C_{2}=p^{2} k / 2 b_{1}=-a_{1} / b_{1}
$$

Using Equation (37) in Equation (34), one has

$$
T(t)=e^{a_{1} t}\left[\cos b_{1} t+\left(-a_{1} / b_{1}\right) \sin b_{1} t\right]
$$

Thus deflection $w$ may be expressed by using Equation (38) and Equation (28) in Equation (3)

$$
\begin{aligned}
w= & {\left[X Y \frac{a}{b}(1-X)\left(1-Y \frac{a}{b}\right)\right]^{2} } \\
& {\left[1+\left(-\frac{b_{11}}{b_{12}}\right) X Y\left(\frac{a}{b}\right)(1-X)\left(1-Y \frac{a}{b}\right)\right] } \\
& \times e^{a_{1} t}\left[\cos b_{1} t+\left(-a_{1} / b_{1}\right) \sin b_{1} t\right]
\end{aligned}
$$

Time period of the vibration of the plate is given by

$$
K=2 \pi / P
$$

where $p$ is the frequency given by Equation (27). 


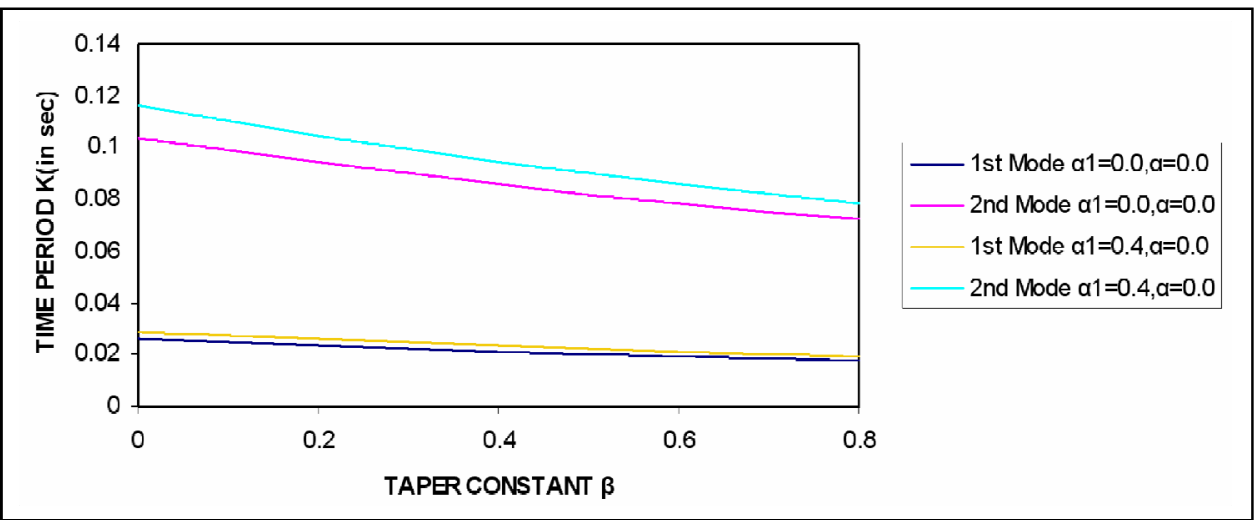

Figure 1. Variation of time period with taper constant of visco-elastic non homogeneous rectangular plate of linearly varying thickness.

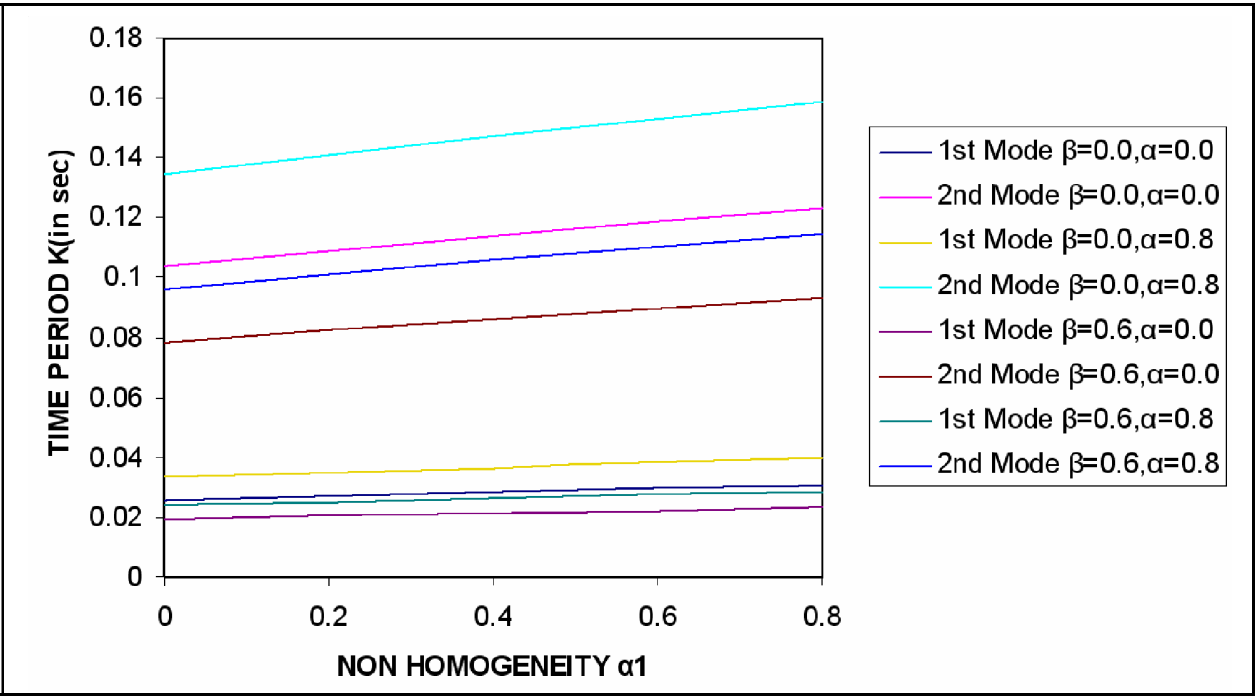

Figure 2. Variation of time period with non homogeneity constant of visco-elastic non homogeneous rectangular plate of linearly varying thickness.

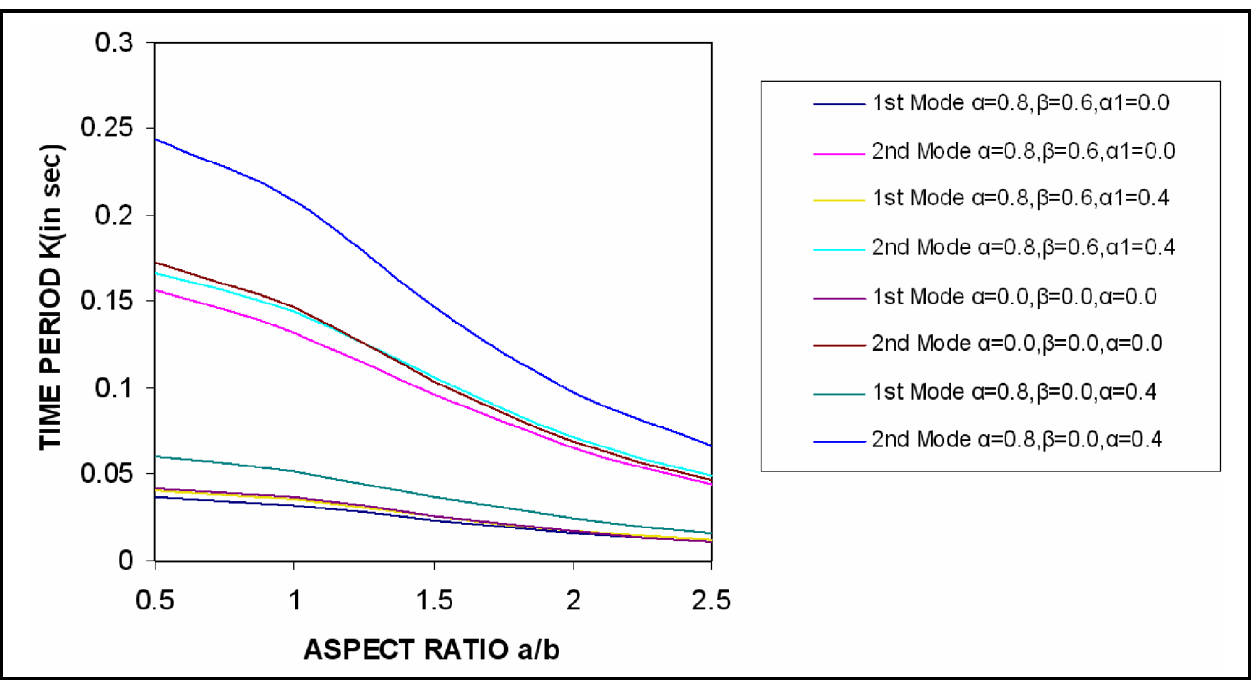

Figure 3. Variation of time period with aspect ratio of visco-elastic non homogeneous rectangular plate of linearly varying thickness. 


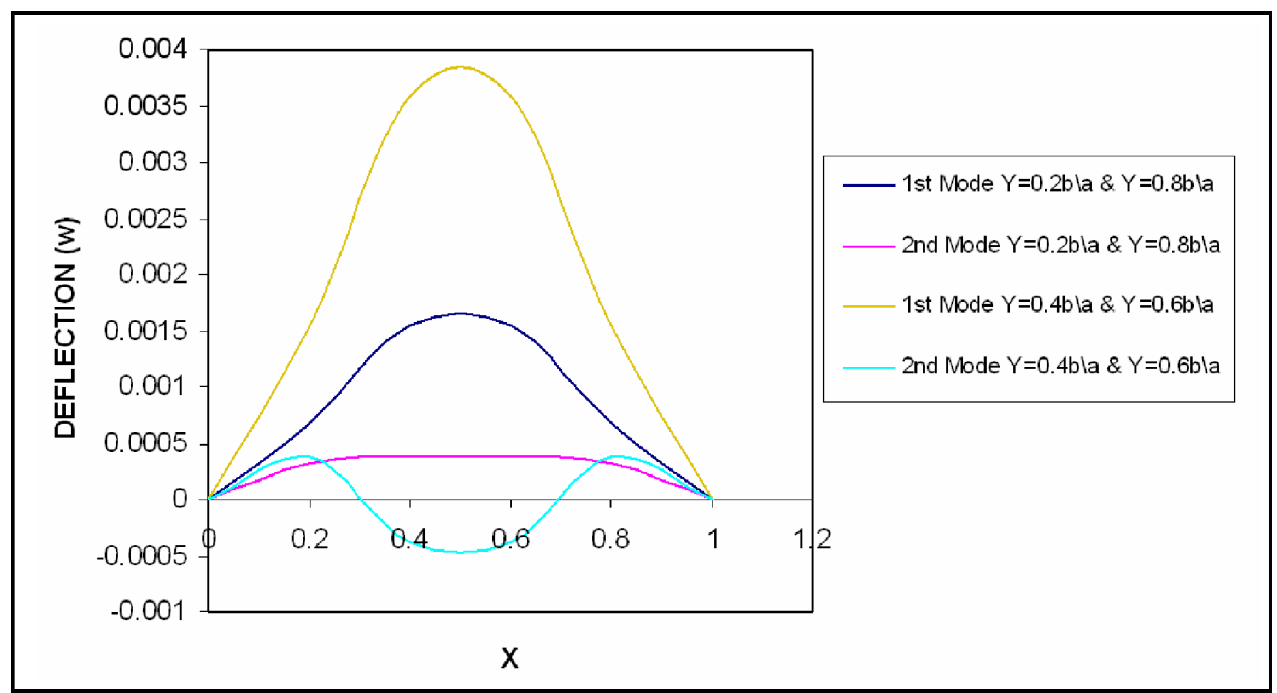

Figure 4. Transverse deflection w Vs $X$ of visco-elastic non homogeneous rectangular plate of linearly varying thickness at initial time $0 . \mathrm{K}$ having constants combination as $\alpha=0.0, \beta=0.0, \alpha_{1}=0.0, \alpha_{4}=0.3, \alpha_{5}=0.2$.

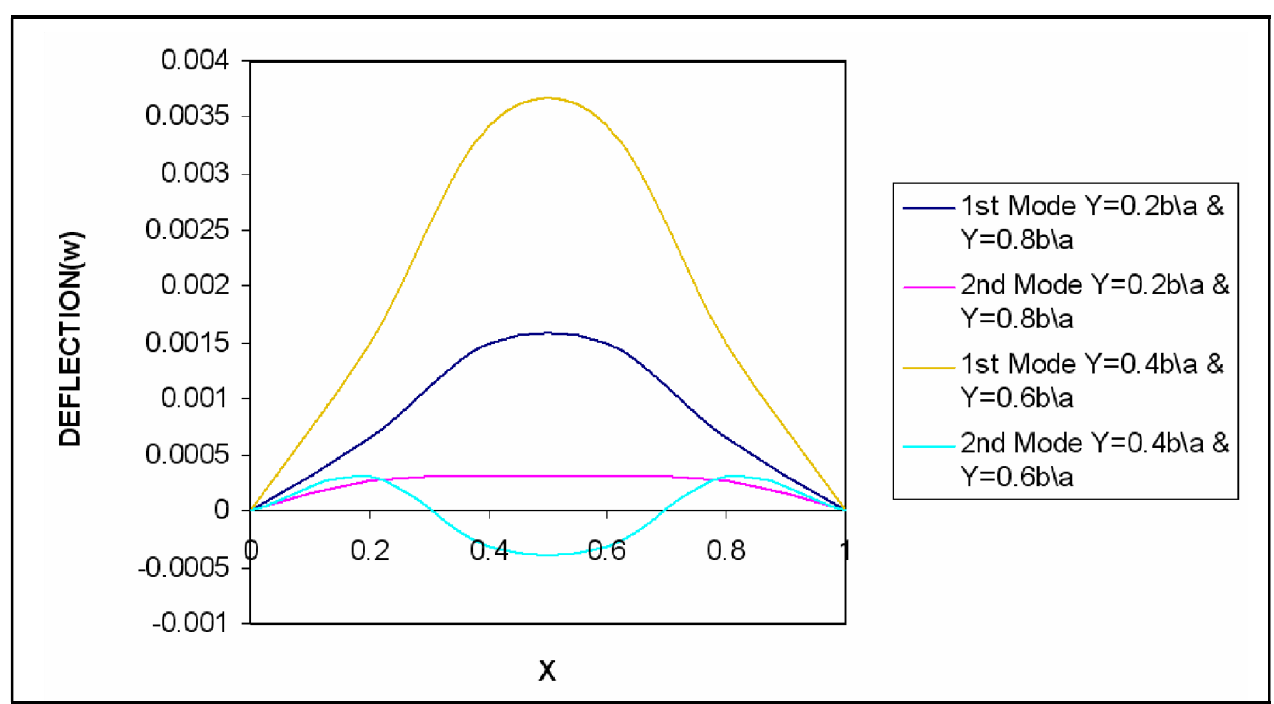

Figure 5. Transverse deflection $w \mathrm{Vs} X$ of visco-elastic non homogeneous rectangular plate of linearly varying thickness at initial time $0 . \mathrm{K}$ having constants combination as $\alpha=0.0, \beta=0.6, \alpha_{1}=0.0, \alpha_{4}=0.3, \alpha_{5}=0.2$.

\section{Results and Discussions}

The orthotropic material parameters have been taken as [3]

$$
\begin{gathered}
E_{2}^{*} / E_{1}^{*}=0.32 \\
E^{*} / E_{1}^{*}=0.04 \\
G_{o} / E_{1}^{*}=0.09 \\
\eta_{o} / G_{o}=0.000069
\end{gathered}
$$

$h=0.01$ (plate thickness)

$\rho_{o}=3 \times 10^{5}$ (mass density per unit volume of the plate material) for calculating the values of this period $\mathrm{K}$ and deflecttion $\mathrm{w}$ for a orthotropic visco-elastic rectangular plate for different values of taper constant $\beta$, thermal constant $(\alpha$, $\alpha_{4}, \alpha_{5}$ ), non homogeneity constant $\alpha_{1}$ and aspect ratio a/b at different points for first two modes of vibrations.

Figure 1 shows the result of time period $\mathrm{K}$ for different values of taper constant $\beta$ and fixed thermal constant $\alpha=0$ and aspect ratio $\mathrm{a} / \mathrm{b}=1.5$ for two values of non homogeneity constant $\alpha_{1}$ are 0.0 and 0.4 for first two modes of vibration. It can be seen that time period $(\mathrm{K})$ decreases when taper constant $(\beta)$ increases for first two modes of vibration.

Figure 2 shows the result of time period $\mathrm{K}$ for first two modes of vibration for different values of non-ho- 
mogeneity constant $\alpha_{1}$ and fixed aspect ratio $\mathrm{a} / \mathrm{b}=1.5$ and four combinations of taper constant $\beta$ and thermal constant $\alpha$ are

$$
\begin{aligned}
& \beta=0.0, \alpha=0.0 \\
& \beta=0.0, \alpha=0.8 \\
& \beta=0.6, \alpha=0.0 \\
& \beta=0.6, \alpha=0.8
\end{aligned}
$$

It can be seen that time period $\mathrm{K}$ increases when nonhomogeneity constant increases for first two modes of vibration.

Figure 3 shows the result of time period $\mathrm{K}$ for different aspect ratio and four combinations of thermal con- stant $\alpha$, taper constant $\beta$ and non-homogeneity constant $\alpha_{1}$ i.e.

$$
\begin{aligned}
& \alpha=0.8, \beta=0.6, \alpha_{1}=0.0 \\
& \alpha=0.8, \beta=0.6, \alpha_{1}=0.4 \\
& \alpha=0.0, \beta=0.0, \alpha_{1}=0.0 \\
& \alpha=0.8, \beta=0.0, \alpha_{1}=0.4
\end{aligned}
$$

It can be seen that time period $\mathrm{K}$ decreases when aspect ratio increases for first two modes of vibration.

Figures 4-7 show the result of deflection for first two modes of vibration for different $\mathrm{X}, \mathrm{Y}$ and fixed aspect ratio $\mathrm{a} / \mathrm{b}=1.5$ for initial time $0 . \mathrm{K}$ and $5 . \mathrm{K}$ for the following combination of thermal constants $\left(\alpha, \alpha_{4}, \alpha_{5}\right)$,

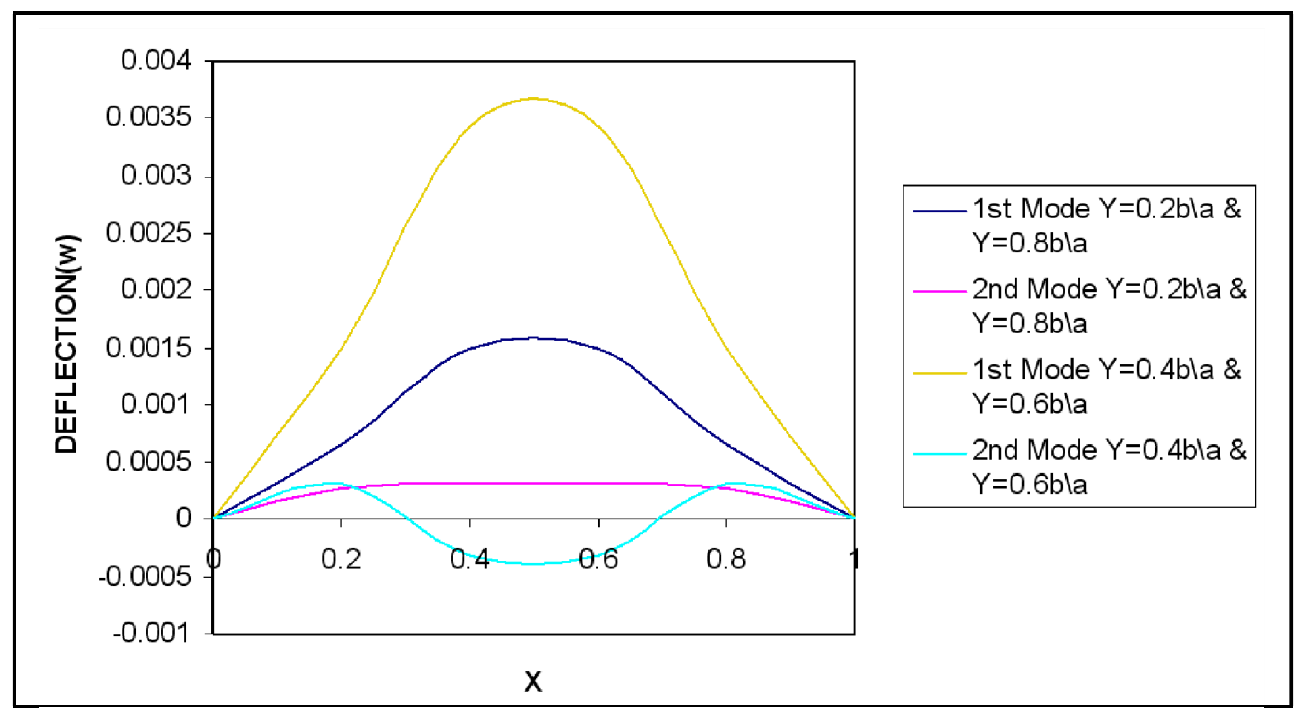

Figure 6. Transverse deflection $\mathrm{w}$ Vs $\mathrm{X}$ of visco-elastic non homogeneous rectangular plate of linearly varying thickness at time 5.K having constants combination as $\alpha=0.8, \beta=0.0, \alpha_{1}=0.4, \alpha_{4}=0.3, \alpha_{5}=0.2$.

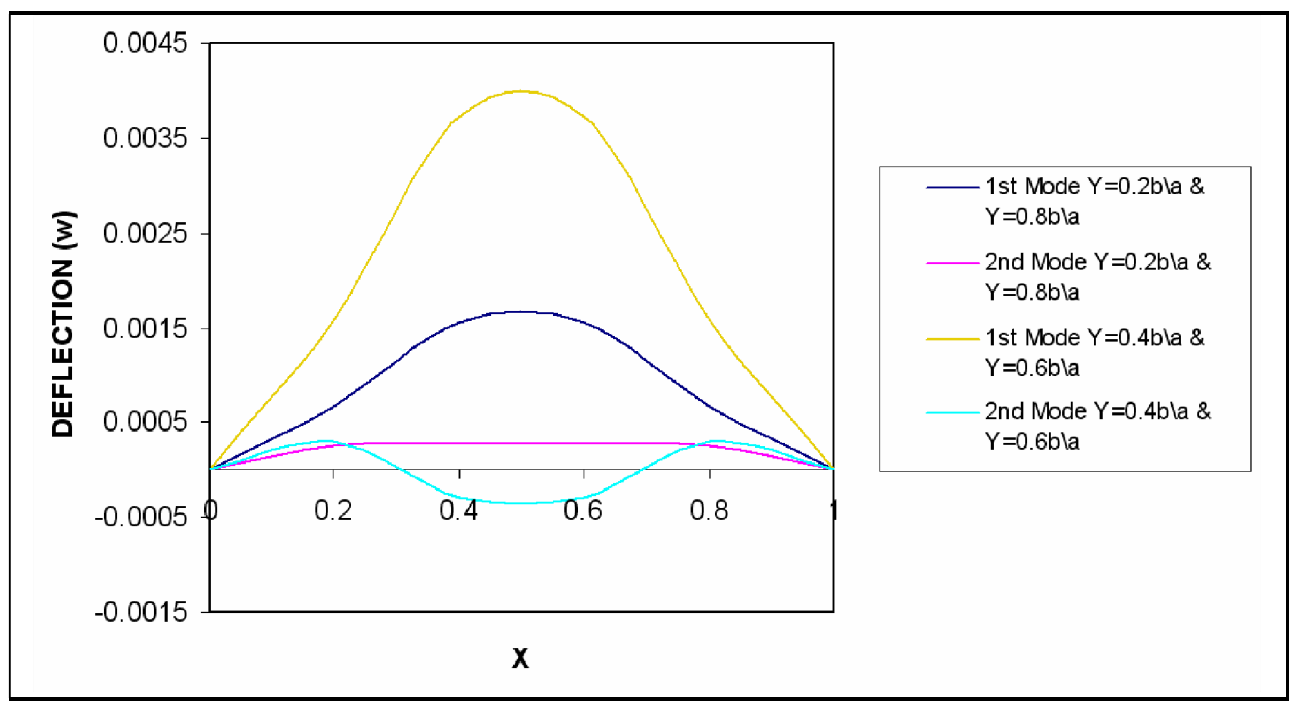

Figure 7. Transverse deflection w Vs $\mathrm{X}$ of visco-elastic non homogeneous rectangular plate of linearly varying thickness at time 5.K having constants combination as $\alpha=0.8, \beta=0.6, \alpha_{1}=0.0, \alpha_{4}=0.3, \alpha_{5}=0.2$. 
taper constant $\beta$ and non-homogeneity constant $\alpha_{1}$.

Results are compared with isotropic plate [12] and found to be in very close agreement.

\section{References}

[1] Z. Sobotka, "Free Vibration of Visco-Elastic Orthotropic Rectangular Plates," Acta Technica, Vol. 23, No. 6, 1978, pp. 678-705.

[2] A. K. Gupta and A. Khanna, "Vibration of Viscoelastic Rectangular Plate with Linearly Thickness Variations in Both Directions," Journal of Sound and Vibration, Vol. 301, No. 3-5, 2007, pp. 450-457.

[3] A. W. Leissa, "Vibration of Plate," NASA SP-60, 1969.

[4] S. R. Li and Y. H. Zhou, "Shooting Method for Non Linear Vibration and Thermal Buckling of Heated Orthotropic Circular Plates," Journal of Sound and Vibration, Vol. 248, No. 2, 2001, pp. 379-386.

[5] D. V. Bambill, C. A. Rossil, P. A. A. Laura and R. E. Rossi, "Transverse Vibrations of an Orthotropic Rectangular Plate of Linearly Varying Thickness and with a Free Edge," Journal of Sound and Vibration, Vol. 235, No. 3, 2000, pp. 530-538.

[6] J. S. Tomar and A. K. Gupta, "Effect of Thermal Gradient on Frequencies of Orthotropic Rectangular Plate Whose Thickness Varies in Two Directions," Journal of Sound and Vibration, Vol. 98, No. 2, 1985, pp. 257-262.

[7] J. S. Tomar and A. K. Gupta, "Thermal Effect on Frequencies of an Orthotropic Rectangular Plate of Linearly Varying Thickness," Journal of Sound and Vibration, Vol. 90, No. 3, 1983, pp. 325-331.

[8] J. S. Tomar and A. K. Gupta, "Effect of Exponential Temperature Variation on Frequencies of an Orthotropic Rectangular Plate of Exponentially Varying Thickness," Proceeding of the Workshop on Computer Application in Continum Mechanics, Roorkee, 11-13 March 1986, pp. 183-188.
[9] U. S. Gupta, R. Lal and S. Sharma, "Vibration Analysis of Non-Homogenous Circular Plate of Nonlinear Thickness Variation by Differential Quadrature Method," Journal of Sound and Vibration, Vol. 298, No. 4-5, 2006, pp. 892 -906 .

[10] A. K. Gupta, T. Johri and R. P. Vats, "Thermal Effect on Vibration of Non-Homogeneous Orthotropic Rectangular Plate Having Bi-Directional Parabolically Varying Thickness," Proceeding of International Conference in World Congress on Engineering and Computer Science 2007 (WCECS 2007), San Francisco, 24-26 October 2007, pp. 784-787.

[11] A. K. Gupta, A. Kumar and D. V. Gupta, "Vibration of Visco-Elastic Orthotropic Parallelogram Plate with Linearly Thickness Variation," Proceeding of International Conference in World Congress on Engineering and Computer Science 2007 (WCECS 2007), San Francisco, 24-26 October 2007, pp. 800-803.

[12] A. K. Gupta and L. Kumar, "Thermal Effect on Vibration of Non-Homogenous Visco-Elastic Rectangular Plate of Linear Varying Thickness," Meccanica, Vol. 43, No. 1, 2008, pp. 47-54.

[13] A. K. Gupta, A. Khanna and D. V. Gupta, "Free Vibration of Clamped Visco-Elastic Rectangular Plate Having BiDirectional Exponentially Thickness Variations," Journal of Theotrocial and Applied Mechanics, Vol. 47, No. 2, 2009, pp. 457-471.

[14] A. K. Gupta, N. Aggarwal, D. V. Gupta, S. Kumar and P. Sharma, "Study of Non-Homogeneity on Free Vibration of Orthotropic Visco-Elastic Rectangular Plate of Parabolic Varying Thickness," Advanced Studies of Theory Physics, Vol. 4, No. 10, 2010, pp. 467-486.

[15] K. Bhasker and B. Kaushik, "Simple and Exact Series Solutions for Flexure of Orthotropic Rectangular Plates with any Combination of Clamped and Simply Supported Edges," Composite Structure, Vol. 63, No. 1, 2004, pp. 63-81. 